\title{
THE BOLOGNA/ESO SEARCH FOR DOUBLE DEGENERATES
}

\author{
Angela Bragaglia, Laura Greggio, and Alvio Renzini \\ Dipartimento di Astronomia, Università di Bologna, CP 596, I-40100 Bologna \\ and \\ Sandro D'Odorico \\ European Southern Observatory, D-8046 Garching b. München
}

In 1984 a program has been set up for a systematic search of double degenerates (DD) among spectroscopically confirmed white dwarfs. For each WD at least two CCD spectra are obtained, which are then cross-correlated in order to get a radial velocity difference, if any. For this purpose we have used the ESO Faint Object Spectrograph and Camera (EFOSC) attached at the ESO $3.6 \mathrm{~m}$ telescope. Exposure times are typically in the range 5-15 min, and the typical error in the radial velocity difference $\left(\Delta v_{\mathbf{r}}\right)$ is $\sim 20 \mathrm{~km} \mathrm{~s}^{-1}$.

Until now we had two observing runs, respectively in September 1985 and January 1988, for a total of 5 usable nights. For 20 WDs we have obtained more than one spectrum. Among them we have singled out two DD candidates. The first object (WD0957-666) has shown $\Delta v_{\mathrm{r}}$ values up to $\sim 220 \mathrm{~km} \mathrm{~s}^{-1}$ and three other spectra were kindly taken for us in April 1988 by Stefano Cristiani. These also showed $\Delta v_{\mathrm{r}}$ 's up to $\sim 140 \mathrm{~km} \mathrm{~s}^{-1}$. Another object (WD0954-710) exhibits a $\Delta v_{\mathrm{r}} \simeq$ $70 \mathrm{~km} \mathrm{~s}^{-1}$. The two DD candidates will be observed again in the next observing run in order to confirm their binarity and in case determine the periods.

Two other objects turned out to be WD+red dwarf pairs whose binary nature was already known from multicolor photometry. One of them (WD0034-211) shows an emission core in all the Balmer lines. For the other (WD0419-487) we have been able to obtain the $\Delta v_{\mathrm{r}}$ 's separately for the WD and the RD components. From them, and from the spectroscopic mass of the WD $\left(0.29 M_{\odot}\right)$ we then estimate the RD to be $\sim 0.15 M_{\odot}$. Subject to the decision of the time allocation committee, we plan to keep searching for DDs and to obtain periods for every candidate pair. More information can be obtained in Bragaglia et al. (The Messenger, 52, 35, 1988). 